\title{
Virtual Assembly Technology on Steel Bridge Members of Bolt Connection
}

\author{
Zhu Hao \\ CCCC Second Harbors Engineering Co., Ltd., Wuhan, Hubei 430040, China \\ CCCC Highway Bridges National Engineering Research Centre CO., Ltd. \\ 15527752@qq.com
}

\begin{abstract}
Keywords: steel bridge members; virtual assembly; steel truss girder; bolted connection; digital measure

Abstract: Trial assembling is an indispensable procedure for the large bridge steel elements, especially for the high strength blot connected steel elements. To replace the traditional assembling method inside the factory, this paper brings out the virtual assembling procedures and method based on the bolt connected bridge, and introduces the typical data measuring method and model establishing, which can be used for complicated elements multi-surface assembling of the steel truss bridge calculation. This technique has already been tested in Hutong Yangtze River Bridge non-navigation span steel structure factory by the means of advanced laser tracking test system. The virtual trial assembling software realized the complicated steel truss girder manufacturing deviation analysis and assembling deviation analysis. After perfecting, the bridge trial assembling inside the factory can be replaced by this method.
\end{abstract}

\section{Introduction}

In recent years, truss bridge has become common in railway bridges and highway and railway combined bridges. With the development of high-speed rail for the past few years, the long-span steel truss bridge has also developed rapidly, such as: Wuhan Tianxingzhou Yangtze River Bridge, Wuhu Yangtze River Bridge, Chongqing Chaotianmen Bridge and Baling River Bridge. The main girder structure of these bridges all adopted truss structure as they all have large quantity of member bars installed and complicated bolting or welding joints. Thus, it is frequently required to conduct trial assembling in the factory during manufacturing process in order to promptly find the unqualified members for timely repair and to ensure safe and orderly construction on the site, especially in high-altitude operation environment. Therefore, trial assembling becomes an important procedure for large steel truss structure, especially high strength blot connected steel member manufacturing.

The trial assembling inside the factory generally requires the same mechanical equipment as that of site construction, sufficiently large field and many laborers, which greatly increases the manufacturing cost and restricts the construction period of project. Even though the level assembling is adopted to replace the three-dimensional trial assembling, its practical effect is barely satisfactory and requires the same amount of investment and time. With virtual pre-assembly technology, this problem can be easily solved. Recently, with the development of modern industrial measurement technology, virtual reality technology, computer aided design technology, etc., the virtual pre-assembly technology has been gradually improved and widely used in bridge engineering at home and abroad.

\section{Virtual pre-assembly technology of bridge members}

The virtual pre-assembly technology applied the virtual assembling technology which has emerged in recent years. From the perspective of product assembly and design, the virtual assembling technology aims to build a multi-mode virtual environment integrating hearing, seeing and touching with the virtual reality technology and computer simulation technology; assisted by input and output equipment of virtual reality, the designer can assemble and plan, test and evaluate the assembly performance of product in a man-machine interactive way in the virtual environment and form an 
economical, reasonable and practical assembly scheme. The virtual assembly technology has become a typical application of virtual reality technology in industrial circle. For steel bridge truss, it means geometric data collection/feature data collection for finished members to be assembled. These data, after input into the computer, are compared with the theoretical coordinate values of the corresponding members input into the computer in advance and the difference is the manufacturing error of the members. With the error precision designated, the computer will determine members having excessive error and manufacturing mistakes and conduct pre-analysis on the problems that may be encountered during installation, and thus to guide the factory in timely repair of problematic members so as to timely identify the inability of installation before the entities are hoisted together. This way, the labor intensity of workers shall be greatly reduced, the productivity can be enhanced and the automatic and intelligent development of production will be furthered.

The virtual pre-assembly technology of bridge members generally includes the following steps: 1) collection of measured data for members, 2) establishment of measured three-dimensional member model or three-dimensional model with measured feature data, 3) pre-assembly of members with reasonable virtual pre-assembly algorithm, 4) result output and three-dimensional visualized display.

\section{Digital measurement technology for virtual pre-assembly of steel bridge members}

The digital measurement technology for virtual pre-assembly of steel bridge members is the most critical step for virtual pre-assembly and the precision of data test can directly affect the pre-assembly results. The trial assembling requirements in Code for Manufacturing of Steel Bridge (Q/CR 9211-2015) are as follows: for welded steel members, the allowable error of span (length) is $\pm 8 \mathrm{~mm}$; for bolt connected members, the bolt hole for main truss of truss girder and bond beam shall $100 \%$ passes through the hole tester which is $0.75 \mathrm{~mm}$ smaller than the designed hole diameter, so the collected virtual pre-assembly data shall have high precision. The following introduction can be used in digital measurement technology for feature data collection of steel bridge members.

\subsection{Digital industrial photogrammetric system}

Digital industrial photogrammetric system refers to the industrial measuring system used in high-precision test of product appearance, which is characterized with high automation, high precision, high efficiency and high flexibility etc. It has been more and more widely used in various fields. For steel structure manufacturing, YOKOGAWA applied the technology in bridge members manufacturing and test [1] and Li Yang of Zhejiang University adopted the imported TRITTOP measuring system to conduct measurement and digital simulation pre-assembly test for steel truss members, which achieved good effect [2]. The overall measurement error of this system can be controlled within $0.02 \mathrm{~mm} / 10 \mathrm{~m}$.

Digital industrial photogrammetric system generally includes high-precision CCD camera, measurement point target, AutoBar, codes and software system. The operation process is: 1) arrange the measurement target, code mark and autobar on the member to be tested (according to different places of tested feature data, the measurement target can be divided into spherical target (testing bolt hole) and L-shaped target (testing edge point), etc.)and the code mark shall satisfy the assembling requirements; 2) the high-precision CCD camera is adopted to shoot pictures of the member and it is required that the code marks on each picture shall be more than two to ensure the assembling precision of later software processing; 3) all the pictures of members to be tested shall be imported into the mature software system that can realize automatic assembling and extract the geometric coordinates of the measurement points; these measurement points can serve as the feature points of pre-assembly measured structure.

\subsection{D laser scanning measurement technologies}

3D laser scanning measurement technology is a full-automatic, high-precision and non-contact stereoscanning technology. It has strong adaptive ability to environment, high data collection 
efficiency and real-time measurement ability. It is not only widely applied in surveying and mapping field, but also developed in engineering, environment detection, urban construction and preservation of cultural relics. In China, Li Chao adopted Leica 3D laser scanner to measure and test the production members of steel structure, which effectively avoided the influence of manufacturing error of steel structure on the installation process and structural safety [3].

3D laser scanning measurement technology tests the members through laser scanner to obtain the point cloud data of complicated steel bridge structure and realize the reconstruction of measured model; high-precision data can be obtained quickly with it, while all-weather operation is also possible. The angle measurement precision of Leica Scan Station P30/40 scanner is 8", ranging precision is $1.2 \mathrm{~mm}+10 \mathrm{ppm}$, the scanning speed can reach 1,000,000 points/second and the target acquisition precision is $2 \mathrm{~mm} @ 50 \mathrm{~m}$.

\subsection{Laser tracker measurement system}

Laser tracker measurement system is a high-precision large-scale measuring instrument in industrial measuring system. It is characterized with high precision, high efficiency, real-time tracking measurement, simple operation, etc. and is widely used in tooling setting in aeronautical manufacture, automobile manufacture and general manufacture [4].

When large-scale complicated steel members are tested by laser tracker measurement system, the spherical target can be used to rapidly measure the bolt hole feature data with high precision. The Leica AT402 rangfinder with the highest measurement precision: ADM has a resolution of $0.1 \mu \mathrm{m}$ and the maximum measurement uncertainty is $10 \mu \mathrm{m}$ and ATR supports continuous tracking and positioning of different reflectors within $320 \mathrm{~m}$ (full range).

\section{Establishment of 3D measured feature model and precision control}

The establishment of 3D measured feature model aims to contrast with the designed theoretical geometric model as well as display the virtual pre-assembly in a visual way. Therefore, the steps for establishing 3D measured feature model are: 1) import the designed 3D theoretical model into the assembly software with the general format of dxf in CAD. Figure 1 shows the 3D model imported into complicated steel truss girder members, 2) mark the places of measurement points in software (the measurement points are numbered automatically), 3) import the geometric coordinates for feature points of digital measurement, 4) correlate and display the measured feature points and theoretical design points by least squares.

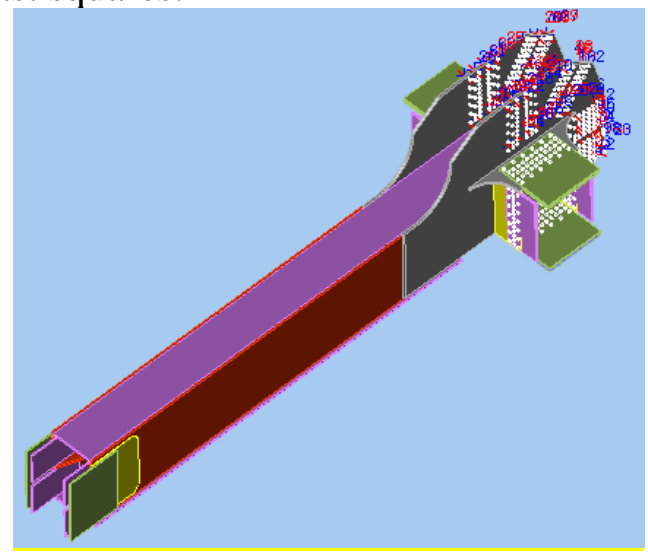

Fig. 13D Model of Steel Truss Girder Members

The automatic correlation and manual correlation can be selected at the time of correlation and after correlation; the precision control can be conducted. The software conducts error comparison between design data and measured data according to the set parameters and automatically calculates the position deviation of feature position while according to color grading display, the output functions such as error statistics output, setting of allowable value and customized statement can also 
be realized. Meanwhile, due to possible different test temperatures of each member bar, the temperature of each member bar shall be corrected to ensure that the test data are not affected by the test temperature.

The 3D measured feature data model of each member bar can be established after the above steps. The single member bar includes such model information as name of member bar, designed 3D model, designed feature points and measured feature points. Classified management will be conducted in software to facilitate later check and pre-assembly.

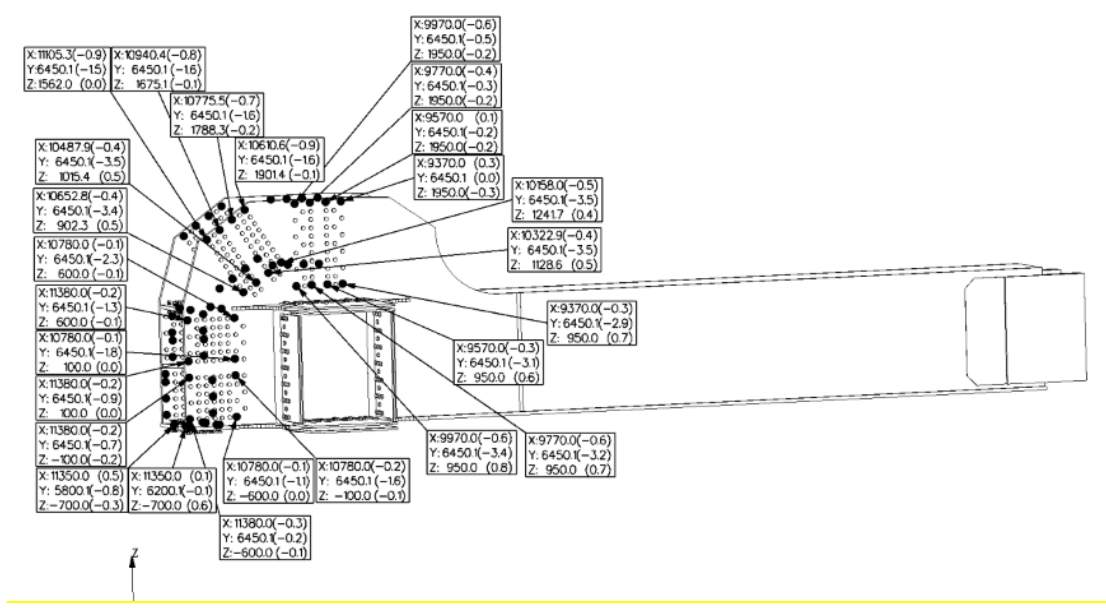

Fig.2 Data Error Display for Measured Feature Point of Bolt Hole of Single Member Bar

\section{Virtual Pre-assembly}

The key to virtual pre-assembly rests in algorithm. With regard to the algorithm for assembling two faces of bolted connection, Italian F.Case ${ }^{[5]}$ first put forth a pre-assembly method based on the bolt hole location and made a brief introduction to this method. Devrim Akcamade the detailed mathematical derivation for the algorithm (analysis of broad-sense spectrum) [6] and conducted the assembly algorithm of two member bars for steel members of bolted connection in Literature [2].

But for the complicated steel truss members, each member bar is connected to many member bars, and the member bar is of box section having four assembling faces; the member bar connection on each assembling face is conducted by one group or more than two groups of assembling plates which cannot be fully resolved with simple algorithm assembling results. In this paper, according to the actual assembly in the factory, the main members shall be first placed in the designed places on jig frame during pre-assembly, and then the assembling plates shall be used for connection. Therefore, the algorithm and assembly steps are improved in the software as follows: first conduct match analysis of the measured value with design value and theoretical value of main member bar (least squares) to make optimal the measured feature value and the theoretical member bar location; then fix the main member bar location; adopt the improved general analysis algorithm in the broad sense, that is to say, do not adjust the feature data of steel truss after match during calculation and only adjust the location of assembling plate; calculate the error of all assembling faces to verify the manufacture precision of member bar.

\section{Engineering application}

Hutong Yangtze River Bridge HTQ-1 Bid Section (non-navigable bridge) is a 23-span 112-meter simply-supported steel truss girder bridge. The main truss adopted three-truss structure, the center-to-center spacing is $2 \times 14.5 \mathrm{~m}$ and the central truss height is $16 \mathrm{~m}$. There are ten panels for each span with the middle panel being $11 \mathrm{~m}$ long and the end panel $10.8 \mathrm{~m}$ long and the high strength bolted connection is adopted.

Three member bars and one assembling plate are selected in the steel structure factory for test with the laser tracker measurement system. The tested member bars and assembling sequence are shown in 
the following Fig. 2 and the virtual pre-assembly software is adopted for error analysis with the results shown in Fig.3. Most assembling errors in directions $\mathrm{X}$ and $\mathrm{Y}$ on bolt plane are within $1 \mathrm{~mm}$ and only individual errors reach $2 \mathrm{~mm}$. The maximum error in axial direction $\mathrm{Z}$ of bolt hole reaches $4 \mathrm{~mm}$ (the influence of error along the axis of bolt hole on the assembly can be neglected). It is proved that the site installation can satisfy the installation requirements.

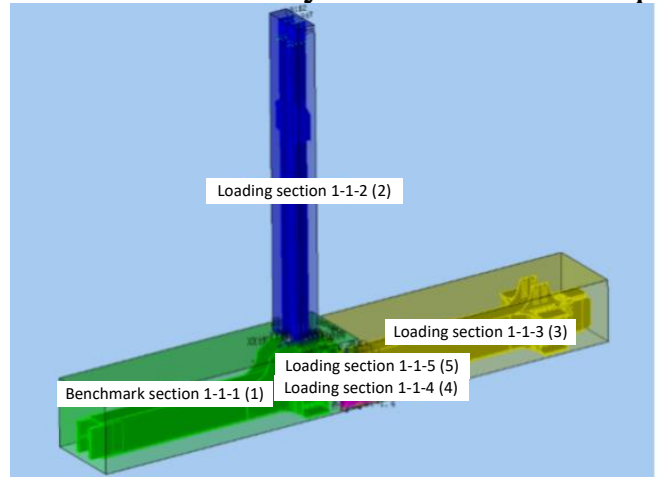

Fig. 3 Tested Member Bar and Pre-assembly Sequence Diagram

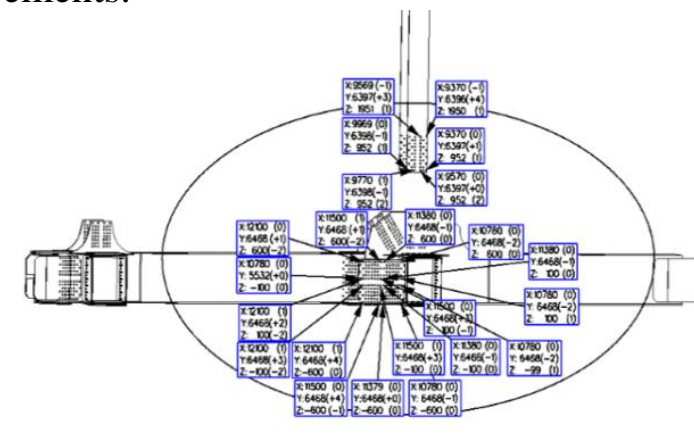

Fig.4 Analysis Diagram for Assembly Error of Complicated Nodes

\section{Conclusion}

This paper introduces the virtual pre-assembling methods based on the bolt connected bridge, including its feature data measurement method and model establishment, which are applicable to the algorithm of multi-face assembling of complicated members for steel truss girder. The test is conducted by the laser tracking test system relying on Hutong Yangtze River Bridge non-navigable bridge and the assembling error analysis is conducted for the complicated steel truss. Through site installation, it is verified that it can be used for the virtual assembly for bridge members of complicated bolted connection and the examination of the processing precision of complicated steel bridge members, especially the special-shaped complicated members and members processed by post hole method. After being improved, this system can be used to replace the bridge trial assembling in factory.

\section{Reference}

[1] Zhang Shanfeng, Guo Zhengxing. New Technologies in Fabrication of Steel Bridge in Japan [J]. World Bridges, 2006, (1): 4-8.

[2] Li Yang. Study of Virtual Trial Assembly Methods of Steel Structure [D]. Zhejiang University, 2015.

[3] Li Chao. Application of Leica 3D Laser Scanning Technology in Steel Structure Test [J] Bulletin of Surveying and Mapping, 2013, (3): 116-117.

[4] Zhang Yifei. Study on Rapid Tracking and Measurement of Laser Tracker [D]. Harbin Institute of Technology, 2015.

[5] Case F,Beinat A,Crosilla F,et al.Virtual trial assembly of a complex steel structure by Generalized Procrustes Analysis Techniques[J].Automation in Construction,2014:155-165.

[6] ETH, Swiss Federal Institute of Technology Zurich, Institute of Geodesy and Photogrammetry. Generalized Procrustes analysis and its applications in photogrammetry [J]. 2003. 